\title{
Características fermentativas, consumo voluntario y digestibilidad de nutrimentos de henilaje de sorgo forrajero [Sorghum bicolor (L.) Moench.] y sorgo-sudan (Sudax) fermentado en pacas cilíndricas ${ }^{1}$
}

\author{
Abner A. Rodríguez ${ }^{2 *}$, Wandaliz Rodríguez-Santana ${ }^{3}$ \\ y Paul F. Randel ${ }^{4}$
}

J. Agric. Univ. P.R. 96(1-2):89-105 (2012)

\begin{abstract}
RESUMEN
Se estudió las características fermentativas, el consumo voluntario y la digestibilidad de sorgo forrajero (SF; Sorghum bicolor) y Sudax (SX; híbrido de sorgo $x$ sudan) conservado como henilaje en pacas cilíndricas. Para determinar las características fermentativas del henilaje, se tomaron muestras de forraje fresco y henilado de dos pacas cilíndricas de cada variedad después de cuatro períodos de fermentación $(3,7,14$ y $30 \mathrm{~d})$. Se analizaron las mismas para $\mathrm{pH}$, concentración de ácidos orgánicos y la relación $\mathrm{N}-\mathrm{NH} 3 / \mathrm{N}$-total. Los datos se analizaron según el diseño experimental completamente aleatorizado (DCA) con arreglo factorial de 2 (variedades de sorgo; SX y SF) x 5 (días de fermentación) con dos repeticiones por combinación. Para determinar el consumo voluntario (CV) y digestibilidad de la materia seca (MS), proteína bruta (PB) y fibra detergente neutro (FDN) se utilizaron ocho ovinos criollos en una prueba de alimentación. Los $15 \mathrm{~d}$ de la prueba fueron distribuidos entre $3 \mathrm{~d}$ de adaptación a las facilidades físicas, $7 \mathrm{~d}$ de adaptación a la dieta experimental y $5 \mathrm{~d}$ de recolección de datos comparativos. A cada ovino se le ofreció diariamente el henilaje correspondiente procedente de dos pacas cilíndricas, a razón de $3 \%$ del peso vivo (PV) a base seca y agua ad libitum. Los datos comparativos se sometieron a un análisis de varianza aplicable a medidas repetidas siendo estas las de los días 1 al 5 de recolección fecal. La composición química a base seca del material vegetativo fresco destinado a henilaje de ambos sorgos (MS de 35 o $36 \%$ ) fue similar con excepción del porcentaje (\%) de PB ( $\mathrm{SX}=5.75$; $\mathrm{SF}=9.05$ ). No hubo diferencias significativas entre las variedades de sorgo en el $\mathrm{pH}$ ni en la concentración de ácidos orgánicos (láctico, acético, propiónico y butírico), ni interacciones significativas entre la variedad y el día de fermentación. El pH en ambas variedades no alcanzó el nivel recomendado (menor de 5) y se observó cierta acumulación
\end{abstract}

${ }^{1}$ Manuscrito sometido el 19 de abril de 2012.

${ }^{2}$ Catedrático, Departamento de Industria Pecuaria, Universidad de Puerto Rico, Recinto Universitario de Mayagüez, Box 9000, Mayagüez, P.R. 00681.

${ }^{3}$ Ex Estudiante Graduada, Departamento de Industria Pecuaria.

${ }^{4}$ Catedrático, Departamento de Industria Pecuaria. 
de amoníaco, lo que indica una fermentación inestable. Hubo diferencias significativas $(P=0.05)$ a favor de SF sobre $S X$ en el CV de MS, PB y FDN; también, en la digestibilidad de MS ( $S X=41.92 \%$; SF $=64.90 \%$ ) y PB (SX $=47.09 \%$; SF $=70.68 \%$ ), pero no de FDN. En resumen, al conservarse como henilaje en pacas cilíndricas, las dos variedades de sorgo mostraron características fermentativas pobres, con valores de $\mathrm{pH}$ mayores de 5 y poca producción de ácido láctico. Las ventajas en $\mathrm{CV}$ y digestibilidad de MS y PB indicaron mayor valor nutritivo de SF que de SX.

Palabras clave: sorgo, henilaje, pacas cilíndricas, fermentación, consumo, digestibilidad

\begin{abstract}
Fermentation characteristics, voluntary intake and nutrient digestibility of forage sorghum [Sorghum bicolor (L.) Moench.] and sorgo-sudan

(Sudax) haylage fermented in round bales
\end{abstract}

The objectives of this study were to determine the fermentative characteristics of forage sorghum (FS; Sorghum bicolor) and Sudax (SX; sorghum $x$ sudan hybrid) preserved as haylage in large round bales (LRB), and to compare the voluntary intake (VI) and in vivo digestibility of haylage of the two varieties. For the first objective, samples taken from two LRB of each sorghum variety after four periods of fermentation $(3,7,14$ and $30 \mathrm{~d})$ plus the initial forage (d 0) were analyzed for $\mathrm{pH}$, concentration of organic acids and $\mathrm{NH}_{3}-\mathrm{N} /$ total $\mathrm{N}$ ratio. The data were analyzed in accordance with a completely randomized experimental design with a factorial arrangement of 2 (sorghum varieties; FS and $\mathrm{SX}$ ) $\mathrm{5}$ (days of fermentation) and two replicates per combination. For determination of VI and digestibility of the dry matter (DM), crude protein (CP) and neutral detergent fiber (NDF) fractions, eight creole sheep (randomly allotted to eight cages and to one of two dietary treatments) were used in a trial of 15-d duration: $3 \mathrm{~d}$ for adaptation to the physical facilities, $7 \mathrm{~d}$ for adaptation to the experimental diet, and $5 \mathrm{~d}$ for collection of comparative data. The daily offering of haylage, taken from two bales of each variety, was at the rate of $3 \%$ of bodyweight on a dry basis, and water was available ad libitum. The comparative data were submitted to analysis of variance applicable to repeated measures; these were days 1 to 5 of fecal collection. The initial chemical composition (dry basis) of the fresh vegetative material ( 35 or $36 \%$ DM) of both sorghums destined to haylage was similar except for $\mathrm{CP}$ percentage $(\mathrm{SX}=5.75$; $\mathrm{SF}=9.05$ ). There were no significant differences between sorghum varieties in $\mathrm{pH}$ or in concentration of organic acids (lactic, acetic, propionic and butyric) of the fermented vegetative material nor significant interactions of variety by day of fermentation. Neither variety reached the recommended $\mathrm{pH}$ level (less than 5), and some accumulation of ammonia was observed indicating an unstable fermentation. There were significant differences ( $P$ $=0.05$ ) in favor of SF over SX in VI of DM, CP and NDF; and in digestibility (\%) of DM (SX $=41.92$; SF $=64.90)$ and $\mathrm{CP}(\mathrm{SX}=47.09$; $\mathrm{SF}=70.68)$, but not of NDF. In summary, poor fermentation characteristics were observed in haylage of SF and SX fermented in LRB. Superiority in DM and CP and digestibility indicated greater nutritive value in FS than in SX.

Key words: sorghum, haylage, round bales, fermentation, intake, digestibility 


\section{INTRODUCCIÓN}

En los últimos años ha surgido un gran interés entre los productores pecuarios en la utilización de ensilaje de sorgo. La planta de sorgo se presta muy bien para la producción de ensilaje debido a su alto contenido de azúcares, que promueve fermentaciones deseables durante su conservación (Miller, 1984). Actualmente predomina el henilaje utilizando pacas cilíndricas como método de conservación de forrajes entre los productores locales. La producción local de henilaje involucra mayormente gramíneas tropicales naturalizadas (GTN). Estas son especies forrajeras con bajos contenidos de carbohidratos solubles en agua (CSA), lo que genera características de ensilaje poco deseables o no satisfactorias, evidenciado por su alto pH y bajo contenido de ácido láctico (González y Rodríguez, 2002; Rodríguez, 1996). Además, pueden ocurrir condiciones propicias para una alta incidencia de bacterias del género Clostridia, microorganismos que ocasionan fermentaciones secundarias, lo que resulta en una pobre aceptabilidad por el animal del material vegetativo fermentado. A diferencia del ensilado de GTN (i.e., hierba guinea, pangola) existe la opción de otras gramíneas con mayor contenido de CSA (i.e., maíz, sorgo) cuya fermentación resulta en $\mathrm{pH}$ más bajo y mayores contenidos de ácido láctico (Martínez-Fleitas, 1998; Arias-Carrasquillo, 1998; Rodríguez, 1996). Intentos más recientes de henilar gramíneas o leguminosas tropicales en pacas cilíndricas han resultado poco exitosos. González y Rodríguez (2002) sometieron GTN a dicho proceso durante 25 y 53 días y obtuvieron fermentaciones poco estables y susceptibles a deterioro aeróbico, observándose valores de $\mathrm{pH}$ mayores de 5.0 y concentraciones de ácido láctico menores a lo deseado. Vázquez-Ortiz (2009) no obtuvo una fermentación láctica al intentar henilar la leguminosa Stylosanthes guianenesis en pacas cilíndricas durante 30 y 72 días de fermentación. No se han estudiado las características fermentativas de forrajes tropicales con mayores contenidos de CSA (i.e., maíz, sorgo) en pacas cilíndricas. Esta investigación se diseñó para semejante determinación y la del consumo voluntario (CV) y la digestibilidad de nutrimentos en dos variedades de sorgo, forrajero (SF) y sorgo-Sudan (Sudax) (SX), preservados como henilaje en pacas cilíndricas.

\section{MATERIALES Y MÉTODOS}

\section{Material vegetativo}

El sorgo forrajero [Sorghum bicolor (L.) Moench.] y el Sudax (Sorghum bicolor $\times S$. bicolor var. sudanense) se sembraron mecánicamente en la Estación Experimental Agrícola de la Universidad de Puerto Rico localizada en el municipio de Lajas, en un suelo Vertisol, en un predio con dimen- 
siones de $149 \mathrm{~m}$ por $36 \mathrm{~m}$. El predio fue subdividido en cuatro parcelas iguales de $9 \mathrm{~m}$ de ancho, alternando la siembra de las dos variedades de sorgo. La distancia entre plantas y entre hileras fue de 20 y $45 \mathrm{~cm}$, respectivamente. Se cosechó el material vegetativo a los 90 días de crecimiento, cortándose a una altura de $6 \mathrm{~cm}$ del suelo. Después del corte, se recolectó tres muestras del material vegetativo fresco (día 0 ) de cada forraje para determinar el pH y composición química inicial (Van Soest et al., 1991; AOAC, 1990). El análisis químico consistió en determinar los contenidos de materia seca, MS; materia orgánica, MO; materia inorgánica, MI; proteína bruta, PB; fibra detergente neutro, FDN; fibra detergente ácido, FDA; hemicelulosa, $\mathrm{HC}$ y carbohidratos no fibrosos, CNF.

Después de dos días de marchitamiento en el campo se formaron hileras del material vegetativo y se prepararon 16 pacas cilíndricas con un peso aproximado de $400 \mathrm{~kg}$, ocho de cada especie, cubiertas con plástico de polietileno, mediante el uso de maquinaria especializada (Class Rollant 46 roto cut $^{5}$ ). Las pacas cilíndricas se transportaron posteriormente al Proyecto de Pequeños Rumiantes localizado en la Finca Alzamora del Recinto Universitario de Mayagüez. Se evaluaron sus características fermentativas durante 30 días y posteriormente el consumo y digestibilidad de la MS, PB y FDN del henilaje por ganado ovino.

\section{Proceso de fermentación}

Se utilizaron las ocho pacas cilíndricas de cada sorgo (forrajero o sudanense) para la determinación de los cambios en $\mathrm{pH}$ y productos de fermentación en vías de formación del henilaje. Las pacas de cada especie se asignaron en duplicado a uno de cuatro periodos de fermentación (3, 7, 14 y 30 d). Para el muestreo de cada paca correspondiente a cada variedad y período de fermentación, se recolectaron ocho submuestras utilizando un muestreador de forraje (Master Forage Probe, $5 \mathrm{~cm}$ diámetro, Dairy One Forage Lab, Ithaca, NY). Las ocho submuestras se mezclaron para formar muestras compuestas destinadas a la determinación de $\mathrm{pH}$ y productos de fermentación (ácidos orgánicos y nitrógeno en forma de amoníaco, $\mathrm{N}-\mathrm{NH}_{3}$ ). Para determinar la concentración de iones de hidrógeno $(\mathrm{pH})$ se pesó $50 \mathrm{~g}$ de muestra y se mezcló con $450 \mathrm{ml}$ de agua deionizada. La mezcla se homogenizó por dos minutos utilizando el equipo Stomacher 3500 (Seward) y se sometió a filtración a través de una tela de tejido abierto. El efluente colectado $(50 \mathrm{ml})$ sin residuos sólidos fue utilizado para la medición del $\mathrm{pH}$ con

\footnotetext{
${ }^{5}$ Los nombres de compañías y de marcas registradas sólo se utilizan para proveer información específica y su uso no constituye garantía por parte de la Estación Experimental Agrícola de la Universidad de Puerto Rico, ni endoso sobre otros productos o equipo que no se mencionan.
} 
un electrodo de combinación (pH/lon 510, Eutech Instruments/Oakton Instruments) estandarizado con soluciones amortiguadoras comerciales de pH 4, 7 y 10 (Fisher Scientific). El efluente se centrifugó por 15 min para su clarificación y se envió al laboratorio comercial (Dairy One Forage Lab., Ithaca, NY) para la determinación de los productos de fermentación (ácidos grasos volátiles: acético, propiónico y butírico; ácido láctico; y $\mathrm{N}-\mathrm{NH}_{3}$ ). Los datos referentes a $\mathrm{pH}$ y productos de fermentación de etapas sucesivas del henilaje de sorgo en formación se analizaron mediante un diseño completamente aleatorizado con un arreglo factorial de 2 (variedades de sorgo; SX y SF) $\times 5$ (días de fermentación: $0,3,7,14$ y 30) con dos repeticiones por combinación. Se realizó un análisis de varianza (ANOVA) junto a la prueba de separación de medias Tukey, utilizando el programa estadístico SAS (SAS Inst., 1990).

Prueba de consumo voluntario y digestibilidad aparente

Para evaluar el consumo y digestibilidad aparente de la MS, PB y FDN de los henilajes se utilizaron ocho ovinos criollos castrados, cuatro por cada variedad de sorgo con un tamaño corporal bastante uniforme (peso vivo, $\mathrm{PV}=28.5 \mathrm{~kg}$ ). Los animales fueron esquilados y desparasitados (Levasol- $2 \mathrm{ml} \times 45.36 \mathrm{~kg}$ PV) antes de comenzar la prueba de alimentación. Esta tuvo una duración de 15 d, los cuales se distribuyeron en tres días de adaptación a las facilidades físicas mientras se alimentó con heno de GTN (mezclas de hierba guinea, Panicum maximum; hierba pangola, Digitaria decumbens; y hierba Johnson, Johnson halapense); siete días de adaptación a la dieta experimental; y cinco días de recolección de datos comparativos. Durante la prueba de alimentación el henilaje ofrecido se trozó mecánicamente a un tamaño de partícula de aproximadamente $10 \mathrm{~cm}$ para facilitar el consumo. Los ovinos fueron distribuidos aleatoriamente entre ocho corrales individuales, con medidas de $1.11 \times 0.74 \mathrm{~m}$ ubicados en línea, y dos tratamientos dietéticos. Los animales se pesaron antes del período de adaptación a la dieta y al empezar la recolección de datos para ofrecer el henilaje diariamente a razón de $3 \%$ PV a base seca. Había agua disponible ad libitum en bebederos galvanizados automáticos. Todo el henilaje ofrecido había tenido un periodo de fermentación mínimo de $30 \mathrm{~d}$ y máximo de $45 \mathrm{~d}$. Diariamente a las 8:00 am se pesó el forraje del día anterior dejado sin consumir y el nuevo ofrecimiento. El consumo se determinó diariamente de la cantidad de alimento ofrecido menos el rechazado ajustándose sus valores a base seca. Para determinar la digestibilidad in vivo de la MS y de las fracciones PB y FDN, se recolectaron muestras del forraje ofrecido y rechazado y de las heces fecales previamente pesadas durante los cinco días de recolección de datos comparativos. Durante este perío- 
do, se utilizaron dos pacas cilíndricas frescas por variedad de sorgo. Para facilitar la recolección de heces se usó una bolsa especial recolectora ajustada a cada animal. Se guardaron submuestras del $10 \%$ de la recolección total. Las muestras de henilaje ofrecido y rechazado y las heces se analizaron para contenidos de MS, PB y FDN según la metodología citada anteriormente. Los datos individuales de consumo voluntario y digestibilidad se sometieron a ANOVA aplicable a medidas repetidas, siendo estas las de los días 1 al 5 de recolección de datos comparativos. El diseño experimental fue completamente aleatorizado. Las observaciones tomadas de la misma unidad experimental con el paso de los días están correlacionadas, por lo cual no se analizarán como observaciones independientes. El modelo estadístico que se utilizó para las medidas repetidas considera los efectos de la dieta, el tiempo y su interacción (efectos fijos), el efecto aleatorio de la unidad experimental (animal) y el error experimental (aleatorio) de diferentes observaciones en la misma unidad en diferentes tiempos.

\section{RESULTADOS Y DISCUSIÓN}

\section{Material vegetativo}

En los dos tipos de sorgo, cosechadas a los 90 d de crecimiento, la composición química inicial del material vegetativo fresco antes de fermentar fue similar con excepción del contenido de PB (Cuadro 1). El contenido de compuestos nitrogenados fue 3.3 unidades porcentuales mayor $(\mathrm{P}<0.05)$ en SF que en SX. En ambas variedades, el contenido de MS fue mayor de $30 \%$ pero menor de $40 \%$, humedad

Cuadro 1.-Composición química inicial de Sudax y sorgo forrajero.

\begin{tabular}{lcccc}
\hline & \multicolumn{2}{c}{ Variedad de sorgo } & & \\
\cline { 2 - 3 } Fracción química & & & \\
\cline { 2 - 3 }$(\%)$ & Sudax & Sorgo Forrajero & EEM $^{2}$ & Valor P \\
\hline MS & $36.02^{3}$ & 34.61 & 0.68 & 0.23 \\
MI $^{4}$ & 94.45 & 94.80 & 1.39 & 0.78 \\
$\mathrm{~PB}^{4}$ & 5.55 & 5.21 & 1.39 & 0.78 \\
$\mathrm{FDN}^{4}$ & $5.75 \mathrm{a}$ & $9.05 \mathrm{~b}$ & 0.15 & 0.01 \\
$\mathrm{FDA}^{4}$ & 60.20 & 59.30 & 3.92 & 0.11 \\
$\mathrm{HC}^{4,5}$ & 34.60 & 34.95 & 3.08 & 0.86 \\
$\mathrm{CNF}^{4,6}$ & 25.60 & 24.35 & 3.45 & 0.09 \\
\hline
\end{tabular}

${ }^{1}$ Media de dos repeticiones.

${ }^{2}$ Error estándar de la media.

${ }^{3}$ Medias con diferentes letras en la misma fila muestran diferencias significativas utilizando la prueba de Tukey $(\mathrm{P}<0.05)$.

${ }^{4}$ Base seca.

${ }^{5} \mathrm{Hemicelulosa},{ }^{6} \mathrm{Carbohidratos}$ no fibrosos. 
adecuada para su conservación en forma de ensilaje pero no de henilaje. Los forrajes conservados como ensilaje con un contenido de MS menor de $30 \%$ son susceptibles a fermentaciones secundarias putrifactivas ocasionadas por bacterias del género Clostridium; en cambio, un nivel de MS mayor de $40 \%$ puede reducir la calidad del ensilaje debido a la pérdida de nutrientes por oxidación y crecimiento de hongos, además de que podría relacionarse inversamente con el contenido de CSA (Edwards y McDonald; citado por McDonald et al., 1991). En este experimento el contenido inicial de humedad del material vegetativo utilizado (SX, 63.98\%; SF, 65.39\%) no fue óptimo para su preservación como henilaje, razón por la cual se sometió a un periodo de marchitamiento en el campo durante $48 \mathrm{~h}$ antes de ser empacado en pacas cilíndricas para su fermentación. Ambas variedades presentaron contenidos iniciales de MO y MI característicos de forrajeras cosechadas sin contaminación de suelo. Los contenidos de paredes celulares (FDN) y las otras fracciones fibrosas (FDA y HC) fueron típicos de sorgos cosechados en el trópico (MartínezFleitas, 1998; Rodríguez, 1996). Ambas variedades presentaron un contenido de CNF adecuado para facilitar la fermentación como ensilaje o henilaje. En el sorgo, los CNF, también llamados carbohidratos no estructurales (CNE), fracción química que incluye los CSA, se localizan preferentemente en las raíces y los tallos basales (Botrel y Gomide; citado por Correa et al., 2009), tratándose principalmente de monosacáridos (glucosa y fructosa), disacáridos (sacarosa y maltosa) y polisacáridos como almidones y fructosanas (Smith; citado por Correa et al., 2009). Los CSA presentes en la fracción CNF o CNE actúan principalmente como sustrato energético para los microorganismos activos durante la fermentación que los convierten en ácidos láctico y acético (en menor proporción), lo que resulta en una acidificación del material ensilado (reducción de $\mathrm{pH}$ ), que es necesario para su preservación. En estudios relacionados, Ward et al. (2000) determinaron la composición química de diferentes tipos de sorgo; para el tipo forrajero encontraron un contenido de MS menor (23.06\%) y un contenido de PB mayor $(13.32 \%)$ que lo observado en el presente estudio, mientras que el contenido de FDN (63.60\%) y su fracción ligno-celulósica (38.02\%) fueron similares. Las diferencias en contenidos de humedad y $\mathrm{PB}$ entre los dos estudios se explican por la edad de cosecha de $60 \mathrm{~d}$ del material vegetativo evaluado en aquel caso. El sorgo forrajero utilizado en el presente experimento mostró una composición química que coincide con los resultados de Martínez-Fleitas (1998) y Rodríguez (1996), quienes evaluaron el efecto de aditivos comerciales sobre las características fermentativas de ensilajes de sorgos forrajero y granífero (Sorghum vulgare). 


\section{Características fermentativas}

El pH y las concentraciones de los productos de fermentación analizados a diferentes intervalos del periodo fermentativo de las dos variedades de sorgo en pacas cilíndricas se presentan en el Cuadro 2. No se observaron diferencias significativas $(\mathrm{P}>0.05)$ en el $\mathrm{pH}$ del material vegetativo fermentado atribuible a la variedad de sorgo, ni a la interacción entre la variedad y el día de fermentación $(\mathrm{P}>0.05)$. A través del periodo fermentativo, la acidez, medida por el $\mathrm{pH}$, no alcanzó valores menores de 5.4. La disminución máxima de $\mathrm{pH}$ ocurrió después de $7 \mathrm{~d}$ de fermentación ( $\mathrm{SX}=5.49 ; \mathrm{SF}=5.57$ ). Posteriormente, el $\mathrm{pH}$ volvió a aumentar y después de 30 d de fermentación registró valores mayores de 6. Los cambios erráticos observados en el $\mathrm{pH}$ del henilaje resultante podría ser el resultado de fermentaciones secundarias causadas por microorganismos indeseables, como bacterias tipo aeróbicas, Clostridium tipo sacarolíticas o proteolíticas y levaduras, que utilizan ácido láctico como sustrato. Los valores mínimos de $\mathrm{pH}$ observados en las pacas cilíndricas en este experimento difieren de los recomendados para un henilaje estable y de alta calidad, lo cual es un $\mathrm{pH}$ no mayor de 5.0 (McDonald et al., 1991; Woolford, 1984). Los valores de $\mathrm{pH}$ mayores de 5 son característicos de henilajes con alto contenido de carbohidratos estructurales, bajo contenido de CSA, alta capacidad amortiguadora y dominancia de microorganismos no deseables para la fermentación láctica, como coliformes, hongos, y levaduras (González y Rodríguez, 2002). Si no se obtiene un $\mathrm{pH}$ estable, microorganismos indeseables, como hongos, levaduras, bacterias coliformes, y Clostridium tipo sacarolíticas o proteolíticas, se proliferan fermentando el ácido láctico, carbohidratos solubles residuales y compuestos nitrogenados con formación de ácido butírico y amoníaco, y un aumento en el pH. Los henilajes pobremente fermentados se caracterizan por tener valores de $\mathrm{pH}$ entre 5.0 y 7.0 (McDonald et al., 1991), ya que no reunen las condiciones necesarias para la estabilización efectiva como la producida por las bacterias productoras de ácido láctico (BPAL), sino que dominan otros microorganismos menos acidificantes (González y Rodríguez, 2002). En este experimento no se encontró efecto significativo atribuible a la variedad de sorgo $(P>0.05)$ sobre el contenido de ácidos orgánicos (láctico, acético, propiónico y butírico) en los henilajes en formación. El ácido láctico, que es el producto más deseable de la fermentación y está asociado con henilajes y ensilajes bien conservados (Woolford, 1984; McDonald et al., 1991), alcanzó su mayor concentración después de 14 y $7 \mathrm{~d}$ de fermentación en SF $(0.88 \%)$ y SX $(0.48 \%)$, respectivamente. A lo largo del periodo fermentativo, el contenido de ácido láctico mostró un patrón variable, lo que es representativo de una ecología microbiana heterogénea y poco estable con ninguna dominancia de microorganismos deseables. Además, 
CUADRo 2. - Efecto del día de fermentación sobre el pH y los productos de fermentación de SF y SX henilados en pacas cilindricas.

\begin{tabular}{|c|c|c|c|c|c|c|c|}
\hline \multirow[b]{2}{*}{ Componente } & \multirow[b]{2}{*}{$\begin{array}{c}\text { Día } \\
\text { de fermentación }\end{array}$} & \multicolumn{2}{|c|}{ Variedades de sorgo } & \multicolumn{4}{|c|}{ Probabilidad } \\
\hline & & Sudax $^{1}$ & Sorgo Forrajero ${ }^{1}$ & $\mathrm{EEM}^{2}$ & Var & Día & Var * Día \\
\hline \multirow[t]{4}{*}{$\mathrm{pH}$} & $\begin{array}{l}0 \\
3\end{array}$ & $\begin{array}{l}6.21 \\
6.47\end{array}$ & $\begin{array}{l}6.31 \\
6.03\end{array}$ & 0.01 & 0.16 & 0.01 & 0.18 \\
\hline & 7 & 5.49 & 5.57 & & & & \\
\hline & 14 & 5.99 & 5.72 & & & & \\
\hline & 30 & 6.14 & 6.10 & & & & \\
\hline \multicolumn{8}{|c|}{ Productos de fermentación (\%) } \\
\hline \multirow[t]{4}{*}{ Acido láctico } & $\begin{array}{l}0 \\
3\end{array}$ & $\begin{array}{l}0.00 \\
0.36\end{array}$ & $\begin{array}{l}0.02 \\
0.45\end{array}$ & 0.03 & 0.47 & 0.03 & 0.88 \\
\hline & 7 & 0.48 & 0.31 & & & & \\
\hline & 14 & 0.01 & 0.88 & & & & \\
\hline & 30 & 0.01 & 0.45 & & & & \\
\hline \multirow[t]{4}{*}{ Acido acético } & $\begin{array}{l}0 \\
3\end{array}$ & $\begin{array}{l}0.04 \\
0.17\end{array}$ & $\begin{array}{l}0.05 \\
0.16\end{array}$ & 0.01 & 0.32 & 0.81 & 0.71 \\
\hline & 7 & 0.36 & 0.28 & & & & \\
\hline & 14 & 0.47 & 0.30 & & & & \\
\hline & 30 & 0.39 & 0.39 & & & & \\
\hline \multirow[t]{4}{*}{ Acido propiónico } & $\begin{array}{l}0 \\
3\end{array}$ & $\begin{array}{l}0.01 \\
0.01\end{array}$ & $\begin{array}{l}0.02 \\
0.01\end{array}$ & 0.01 & 0.17 & 0.20 & 0.71 \\
\hline & 7 & 0.09 & 0.03 & & & & \\
\hline & 14 & 0.16 & 0.06 & & & & \\
\hline & 30 & 0.10 & 0.08 & & & & \\
\hline
\end{tabular}


CuADro 2.-(Continued) Efecto del día de fermentación sobre el pH y los productos de fermentación de SF y SX henilados en pacas cilindricas.

\begin{tabular}{|c|c|c|c|c|c|c|c|}
\hline \multirow[t]{4}{*}{ Ácido butírico } & $\begin{array}{l}0 \\
3\end{array}$ & $\begin{array}{l}0.00 \\
0.46\end{array}$ & $\begin{array}{l}0.12 \\
0.21\end{array}$ & \multirow[t]{4}{*}{0.01} & \multirow[t]{4}{*}{0.21} & \multirow[t]{4}{*}{0.72} & \multirow[t]{4}{*}{0.82} \\
\hline & 7 & 0.15 & 0.12 & & & & \\
\hline & 14 & 0.30 & 0.19 & & & & \\
\hline & 30 & 0.23 & 0.19 & & & & \\
\hline \multirow[t]{4}{*}{$\mathrm{N}-\mathrm{NH}_{3} / \mathrm{N}$-total } & $\begin{array}{l}0 \\
3\end{array}$ & $\begin{array}{l}0.54 \\
2.71\end{array}$ & $\begin{array}{l}0.28 \\
0.28\end{array}$ & \multirow[t]{4}{*}{0.74} & \multirow[t]{4}{*}{0.01} & \multirow[t]{4}{*}{0.04} & \multirow[t]{4}{*}{0.11} \\
\hline & 7 & 2.83 & 0.62 & & & & \\
\hline & 14 & 7.66 & 1.17 & & & & \\
\hline & 30 & 3.09 & 2.04 & & & & \\
\hline
\end{tabular}

${ }^{1}$ Media de dos repeticione.

${ }^{2}$ Error estándar de la media. 
el contenido de ácido láctico observado en este experimento fue muy inferior al valor mínimo recomendado para un henilaje estable (1.5\%), lo que es indicativo de una fermentación láctica pobre.

A través del periodo fermentativo se observó un patrón similar al obtenido con ácido láctico con la producción de ácido acético en los henilajes. La concentración del mismo osciló entre 0.04 a $0.47 \%$ en las dos variedades de sorgo, valores que también reflejan poco dominancia de las BPAL tipo heterofermentativas o de bacterias acetogénicas, lo que también contribuye a fermentaciones inadecuadas, poco dinámicas, e inestables. Durante el transcurso de la fermentación, se observó la producción de ácido propiónico pero en cantidades muy pequeñas y sin ningún significancia para la fermentación. La producción de ácido butírico se mantuvo relativamente constante desde los $3 \mathrm{~d}$ hasta los $30 \mathrm{~d}$ de fermentación, lo que podría ser indicativo de fermentaciones secundarias producto de la actividad metabólica de microorganismos no deseables (bacterias aeróbicas del género Clostridium o levaduras que utilizan ácido láctico como sustrato). La utilización de este ácido orgánico está asociada con fermentaciones secundarias que utilizan también carbohidratos residuales y compuestos nitrogenados. La fermentación inestable observada en los henilajes de ambas variedades de sorgo también se refleja en la producción constante de amoníaco después de $3 \mathrm{~d}$ y de $14 \mathrm{~d}$ de fermentación en henilajes de SX y SF, respectivamente. Entre las dos variedades de sorgo se observó una mayor $(\mathrm{P}<0.05)$ relación de $\mathrm{N}-\mathrm{NH}_{3} / \mathrm{N}$-total después de 7, 14 y 30 d en SX que en SF, esto a pesar de que el contenido inicial de $\mathrm{PB}$ en SX fue 3.3 unidades porcentuales menor que en la variedad forrajera. A pesar de que las características fermentativas indican que ninguno de los sorgos henilados en pacas cilíndricas representa un forraje conservado de calidad, es posible inferir (basado en un menor $\mathrm{pH}$ final, un contenido mayor de ácido láctico y menor de ácido butírico y menor hidrólisis de compuestos nitrogenados para producir amoníaco) que la variedad SF presentó mejor aptitud para henilarse que la Sudax. Sin embargo, y ante la similitud de la composición inicial entre las dos variedades, otros factores asociados de la planta (i.e., microorganismos epifíticos, proporción tallo: hoja, contenido de compuestos inhibidores) o del manejo (densidad de compactación del material vegetativo dentro de la paca durante la fermentación) podrían influir en el proceso de henilar. Otras investigaciones locales han obtenido resultados similares al evaluar la producción de henilaje en pacas cilíndricas como método de conservación en GTN (González y Rodríguez, 2002) y leguminosas tropicales (i.e., Stylosanthes guienensis; Vázquez-Ortiz, 2009). En los citados estudios, se observaron fermentaciones pobres con $\mathrm{pH}$ altos, poca producción de ácido láctico y bajas poblaciones de BPAL. Esos autores atribuyeron los resultados a 
los bajos contenidos de CSA presentes en el material vegetativo fresco de las GTN y leguminosas, además de la capacidad amortiguadora de estas últimas. En cambio, las distintas variedades de sorgo presentan contenidos óptimos de CNE para promover la fermentación láctica y bajos o moderados contenidos de PB y otros ácidos orgánicos asociados que aportan a la capacidad amortiguadora de los forrajes o su capacidad de resistir cambios en $\mathrm{pH}$ durante la fermentación (Vázquez-Ortiz, 2009). Las características químicas iniciales favorables del sorgo para conservarse de forma anaeróbica y la pobre fermentación obtenida al henilarlo en pacas cilíndricas hacen inferir que son otras condiciones, como la falta de compactación adecuada, la baja densidad del material al henilarse y una duración prolongada de la fase "lag" de bacterias productoras de ácido láctico durante la fermentación, las que podrían limitar la debida formación de henilaje utilizando el aludido método. Las características físicas de ambas variedades de sorgo evaluadas, como la alta proporción de tallos y el tamaño de partícula relativamente grande del material vegetativo al momento de empacarse, pueden haber impedido la compactación adecuada del mismo, aumentando así la presencia de oxígeno y ocasionando las condiciones para fermentaciones secundarias no deseables. En otros estudios relacionados, también la conservación de forrajes en pacas cilíndricas resultó en fermentaciones inestables, debido posiblemente a la compactación inadecuada del material vegetativo al momento de empacarse (Kung y Stokes, 2001).

Consumo voluntario y digestibilidad aparente de la materia seca y nutrimentos

En este experimento se evaluó el CV y la digestibilidad de la MS, PB y FDN de las dos variedades de sorgo ofrecidas a ovinos en forma de henilaje como dieta única. El contenido porcentual de estas tres fracciones difirió entre los dos henilajes ofrecidos pero no estadísticamente en el caso de la MS. El contenido de MS fue 5.52 unidades porcentuales mayor $(\mathrm{P}=0.05)$ en el henilaje de SX que en el de SF ofrecidos ambos después de 30 d de fermentación (53.48 vs. $47.96 \%$ ). El contenido de $\mathrm{PB}$ fue mayor por 2.07 unidades porcentuales en SF que en SX $(9.07 \%$ vs. $7.00 \%$ ) pero el contenido de paredes celulares fue menor por 4.99 unidades (54.24 vs. 59.23\%). Los valores de PB encontrados en este experimento coinciden con los observados por Balwani (1969) de 7.5\% y $10.1 \%$ en sorgo forrajero y granífero, respectivamente.

Durante la prueba de alimentación con ovinos, la cantidad ofrecida ( $\mathrm{g} / \mathrm{d}$ ) de MS de los dos materiales vegetativos fermentados fue estadísticamente similar; en cambio, las cantidades de PB y FDN ofrecidas variaron conforme a la composición química porcentual de los henilajes (Cuadro 3). El ofrecimiento de PB fue mayor $(\mathrm{P}=0.03)$ y el de FDN (P 
Cuadro 3.-Consumo voluntario y digestibilidad de la MS, PB y FDN de dos variedades de sorgo heniladas en pacas cilindricas.

\begin{tabular}{|c|c|c|c|c|}
\hline \multirow[b]{2}{*}{ Componente } & \multicolumn{2}{|c|}{ Variedad } & \multirow[b]{2}{*}{$\mathrm{EEM}^{1}$} & \multirow[b]{2}{*}{ Probabilidad } \\
\hline & Sudax & Forrajero & & \\
\hline \multicolumn{4}{|c|}{ Alimento ofrecido $(\mathrm{g} / \mathrm{d})$} & 0.17 \\
\hline PB & $81.01 b^{2}$ & $95.00 \mathrm{a}$ & 0.49 & 0.03 \\
\hline FDN & $685.46 \mathrm{a}$ & $569.99 \mathrm{~b}$ & 3.22 & 0.01 \\
\hline \multicolumn{5}{|c|}{ Alimento rechazado (g/d) } \\
\hline $\begin{array}{l}\text { MS } \\
\mathrm{PB}\end{array}$ & $\begin{array}{r}674.59 \mathrm{a} \\
41.78 \mathrm{a}\end{array}$ & $\begin{array}{r}253.82 \mathrm{~b} \\
8.92 \mathrm{~b}\end{array}$ & $\begin{array}{l}6.91 \\
0.52\end{array}$ & $\begin{array}{l}0.01 \\
0.01\end{array}$ \\
\hline FDN & $492.59 \mathrm{a}$ & $195.93 \mathrm{~b}$ & 4.47 & 0.01 \\
\hline \multicolumn{5}{|c|}{ Consumo $(g / d)$} \\
\hline $\begin{array}{l}\text { MS } \\
\text { PB }\end{array}$ & $\begin{array}{r}482.71 \mathrm{~b} \\
39.23 \mathrm{~b}\end{array}$ & $\begin{array}{r}801.72 \mathrm{a} \\
7.17 \mathrm{a}\end{array}$ & $\begin{array}{l}7.87 \\
0.63\end{array}$ & $\begin{array}{l}0.01 \\
0.01\end{array}$ \\
\hline FDN & $192.87 \mathrm{~b}$ & $374.06 \mathrm{a}$ & 5.02 & 0.01 \\
\hline \multicolumn{5}{|c|}{ Digestibilidad (\%) } \\
\hline $\begin{array}{l}\text { MS } \\
\text { PB }\end{array}$ & $\begin{array}{l}41.92 \mathrm{a} \\
47.09 \mathrm{a}\end{array}$ & $\begin{array}{l}64.90 \mathrm{~b} \\
70.68 \mathrm{~b}\end{array}$ & $\begin{array}{l}0.55 \\
0.42\end{array}$ & $\begin{array}{l}0.01 \\
0.01\end{array}$ \\
\hline FDN & $43.87 \mathrm{a}$ & $45.10 \mathrm{~b}$ & 0.97 & 0.90 \\
\hline
\end{tabular}

${ }^{1}$ EEM- Error estándar de la media.

${ }^{2}$ Valores en una hilera con letras diferentes difieren significativamente $(\mathrm{P}<0.05)$.

$=0.01$ ) menor en animales alimentados con SF que con SX. Se observó un mayor rechazo de MS, PB y FDN en ovinos alimentados con SX que con SF. Hubo notables diferencias en el consumo voluntario (g/d) de MS ( 801.7 vs. 482.7$)$, PB (76.2 vs. 39.2$)$ y FDN (374.1 vs. 192.9 ) a favor de SF sobre SX (Cuadro 3).

El CV de los animales está influenciado por las condiciones ambientales, por rasgos inherentes de la planta y propios del animal y por las características organolépticas (olfativas, de sabor, visuales y táctiles) del alimento ofrecido. En este estudio el mayor contenido de ácido butírico y nitrógeno amoniacal, asociado con la casi ausencia de ácido láctico, observado en el henilaje de SX puede haber afectado adversamente el CV del mismo por los ovinos. La presencia de ácido butírico y amoníaco en forrajes conservados húmedos se ha relacionado con una disminución en el CV animal por asociarse con la putrefacción y olor a rancidez (McDonald et al., 1991). La producción de butirato y amoníaco es el resultado de fermentaciones secundarias donde diferentes tipos de microorganismos (i.e., Clostridium) hidrolizan carbohidratos o compuestos nitrogenados. Estos procesos son contrarios a la producción de buenos forrajes conservados. Visualmente también se observó una mayor cantidad de hongos, predominantemente blancos, en el henilaje 
de SX que en el de SF. No se dispone de evidencia para relacionar estos hongos con la producción de toxinas, ni se enumeraron o identificaron en esta investigación, pero los hongos de los géneros Geotrichum y $\mathrm{Mu}$ corales se caracterizan por la formación de colonias de coloración blanca y su presencia en los ensilajes se asocia inversamente con el CV por los animales (Lallemand, Inc., 2002). Este factor pudo ser determinante en el bajo consumo de forraje observado en los ovinos alimentados con henilaje de SX, el cual representó solamente el 1.55\% del PV de los animales en base seca diariamente, contrario al henilaje de SF que fue consumido a razón de $3 \%$ del PV.

En otras investigaciones la conservación de gramíneas tropicales en pacas cilíndricas generalmente ha resultado en fermentaciones pobres, lo cual hace al forraje susceptible al deterioro aeróbico conducente a una disminución en su valor nutritivo. También, este tipo de henilaje está asociado a una baja aceptación animal y bajo CV debido a su alto contenido de amoníaco y de aminas (León-Álamo, 2003; González y Rodríguez, 2002; Harrison y Blauwiekel, 1994). En investigaciones con henilaje de leguminosas tropicales en pacas cilíndricas también se ha visto la presencia de hongos y levaduras en el forraje al momento de su exposición aeróbica, lo que afectó adversamente el CV por ovinos y caprinos (Vázquez-Ortiz, 2009).

Además de un mayor CV del henilaje de SF, en este experimento también se observó mayor digestibilidad de la MS y $\mathrm{PB}$ de $\mathrm{SF}(\mathrm{P}<$ 0.05), pero la digestibilidad de su FDN fue similar a la de SX. Los valores de digestibilidad observados en el henilaje de SF coinciden con los de investigaciones previas para evaluar variedades de sorgo forrajero conservado como ensilaje en rumiantes. Balwani (1969) obtuvo una digestibilidad de la MS de 55\% para sorgo forrajero, valor mayor que el observado en sorgo granífero. Los mismos autores reportaron una digestibilidad de PB de $36 \%$ en sorgo granífero, la cual consideraron sumamente baja para un material vegetativo con un contenido de $\mathrm{PB}$ de $10.1 \%$.

Ward et al. (2000) realizaron un estudio de dos años para determinar la composición y digestibilidad de ensilajes de sorgo forrajero, "pearl millet" y maíz, obteniendo respectivos valores de digestibilidad de la MS de $56.83,51.36$ y $58.13 \%$. En ambos años, se encontraron valores similares para el maíz y el sorgo forrajero, por lo que la decisión de utilizar un cultivo u otro sería a base de otras consideraciones como rendimiento, economía, equipo y maquinaria disponible y condiciones climáticas regionales. Oliver et al. (2004) también evaluaron la digestibilidad de nutrientes de dos híbridos de sorgo forrajero y ensilaje de maíz, y sus resultados concuerdan con los del presente experimento. El sorgo tuvo las digestibilidades: MS, 52.5\%; PB, 51.3\%; y FDN, 40.8\%. 
Las diferencias en coeficiente de digestibilidad entre variedades de sorgo, como el convencional forrajero, el Brown Mid Rib (BMR) e híbridos de sorgo-sudan, se explican por diferencias en la extensión y la digestión de la FDN, siendo estas menores en sorgos no mejorados (Dann et al., 2008).

En los resultados presentes, las digestibilidades de la MS, PB y FDN de SF fueron mayores que las de SX por 22.98, 23.59 y 1.23 unidades porcentuales, respectivamente. Además, se observaron características fermentativas menos deseables y mayor deterioro aeróbico en el henilaje de SX que en el de SF. El bajo consumo de MS y por ende de PB de los ovinos alimentados con SX fermentado puede haber influido negativamente en la digestibilidad de la MS y la de otros nutrimentos. El contenido porcentual inicial de PB del henilaje de sorgo Sudax fue de $7 \%$, valor menor al mínimo requerido teóricamente (8\%) para mantener un funcionamiento ruminal adecuado (NRC, 1985). Este valor marginalmente deficiente asociado a cierto grado de hidrólisis de los compuestos nitrogenados durante el proceso de fermentación podría explicar la baja digestibilidad aparente de la PB y posiblemente, en menor grado, la de paredes celulares en ovinos alimentados con henilaje de SX. Ovinos adultos bajo condiciones de mantenimiento requieren en teoría un promedio de 60 a $100 \mathrm{~g}$ de $\mathrm{PB} / \mathrm{d}$ en dependencia de su PV (NRC, 1985), cantidad muy por encima del consumo observado (39 g/d) en este experimento.

\section{CONCLUSIONES}

$\mathrm{Al}$ conservarse como henilaje en pacas cilíndricas las variedades de sorgo SF y SX, ambas mostraron características fermentativas pobres, caracterizadas por valores de $\mathrm{pH}$ mayores de 5 , y poca producción de ácido láctico. Si bien el SF lució ligeramente mejor en este respecto, las diferencias no fueron estadísticamente significativas. En la prueba in vivo con ovinos hubo diferencias en la digestibilidad de la MS (64.9 vs. $41.9 \%$ ) y de la PB (70.7 vs. $47.1 \%$ ), pero no significativamente en la de FDN (45.1 vs. $43.9 \%$ ); posiblemente estas diferencias sean reflejo de un nivel marginalmente deficiente de $\mathrm{PB}$ en el SX (7.0\%) relativo al SF $(9.07 \%)$ y a mayor desarrollo de hongos observado visualmente en aquel.

\section{LITERATURA CITADA}

AOAC, 1990. Official Methods of Analysis of the Association of Official Analytical Chemists. 15th Ed, Arlington, VA. 
Arias-Carrasquillo, F., 1998. Características fermentativas y estabilidad aeróbica de dos variedades de maíz tropical y hierba guinea ensilada a diferentes estados de madurez. Universidad de Puerto Rico. Recinto Universitario de Mayagüez, Tesis MS.

Balwani, T. L., 1969. Evaluation of green chop and ensiled sorghums, corn silage and perennial forages using digestión trials and VFA production in sheep. J. Anim Sci. 28:90-97.

Correa, H. J., H. Arroyave, Y. Henao, A. López y J. M. Cerón, 2009. Pasto Maralfalfa: Mitos y Realidades (Parte Segunda). Producción Animal. Univ. Nac. de Colombia; Cooperativa COLANTA. http://www.engormix.com/pasto_maralfalfa_mitos_ realidades_s_articulos_440_GDC.htm

Dann, H. M., R. J. Grant, K. W. Cotanch, E. D. Thomas, C. S. Ballard y R. Rice, 2008. Comparison of brown midrib sorghum-sudangrass with corn silage on lactational performance and nutrient digestibility in Holstein dairy cows. J. Dairy Sci. 91:663672.

González, G. y A. A. Rodríguez, 2002. Effect of storage method on fermentation characteristics, aerobic stability, and forage intake of tropical grasses ensiled in round bales. J. Dairy Sci. 86:926-933.

Harrison, J. H. y R. Blauwiekel, 1994. Fermentation and utilization of grass silage. $J$. Dairy Sci. 77:3209-3235.

Kung, L., Jr., y M. R. Stokes, 2001. Analyzing silages for fermentation end products. http://ag.udel.edu/anfs/faculty/kung/articles/analyzing_silages_for_fermentati.htm

Lallemand, Inc., 2002. Silage Management Handbook: A resource to help you maximize the value of your forage. Lallemand Animal Nutrition, Milwaukee, WI.

León-Alamo, F. J., 2003. Consumo voluntario y digestibilidad de nutrientes de heno de gramíneas tropicales nativas y ensilaje de sorgo y el efecto de la suplementación con residuos fermentados de pescadería. Universidad de Puerto Rico, Recinto Universitario de Mayagüez. Tesis M.S.

Martínez-Fleitas, J., 1998. Efecto de la aplicación de aditivos comerciales sobre las características fermentativas y estabilidad aeróbica de forrajeras ensiladas en ambientes tropicales. Universidad de Puerto Rico, Recinto Universitario de Mayagüez. Tesis M.S. pp.15, 28-31.

McDonald, P., A. R. Henderson y S. J. E. Heron, 1991. The Biochemistry of Silage. $2^{\text {nd }}$ Ed. Chalcombe Printers Ltd., Aberystwyth, UK.

Miller, D. A., 1984. Forage Crops. McGraw-Hill Book Company. New York, NY. pp. 486495.

National Research Council, 1985. Nutrient Requirements of Sheep. $6^{\text {th }}$ Ed. National Academy Press. Washington, D.C. pp. 112.

Oliver, A. L., R. J. Grant, J. F. Pedersen y J. O'Rear, 2004. Comparison of brown midrib-6 and -18 forage sorghum with conventional sorghum and corn silage in diets of lactating dairy cows. J. Dairy Sci. 87:637-644.

Rodríguez, A. A., 1996. Studies on the efficacy of a homofermentative lactic acid producing bacterial inoculants and commercial, plant cell wall- degrading enzyme mixtures to enhance the fermentation characteristics and aerobic stability of forages ensiled in temperate and tropical environments. Michigan State University. $\mathrm{PhD}$ dissertation. pp.35

SAS Inst., 1990. SAS/STAT® User's Guide (Release 6.12). SAS Inst., Inc., Cary, NC.

Van Soest, P. J., J. B. Robertson y B. A. Lewis, 1991. Methods for dietary fiber, neutral detergent fiber, and non-starch polysaccarides in relation to animal nutrition. $J$. Dairy Sci. 74:473-481. 
Vázquez-Ortiz, M., 2009. Características fermentativas, estabilidad aeróbica, consumo voluntario y degradabilidad in vitro de henilaje de Stylosanthes guianensis. Universidad de Puerto Rico, Recinto Universitario de Mayagüez. Tesis M.S. pp.16,28,42

Ward, J. D., D. D. Redfearn, M. E. McCormick y G. J. Cuomo, 2000. Chemical composition, ensiling characteristics, and apparent digestibility of summer annual forages in a subtropical double-cropping system with annual ryegrass. J. Dairy Sci. 84:177182.

Woolford, M. K., 1984. The Silage Fermentation. Microbiological Series, No.14. Marcel Dekker: New York, and Basle. 